\title{
ESTIMATION OF FATIGUE PROPERTIES OF COMPOSITE STRUCTURES
}

\author{
Miroslaw Rodzewicz \\ Warsaw University of Technology, Warsaw, Poland
}

\begin{abstract}
The paper presents the proposal of a simplified method of fatigue properties estimation for polymer composite structures.
\end{abstract}

\section{INTRODUCTION}

For fatigue life evaluation of aircraft or sailplane structures, the data regarding the load spectrum and fatigue (cyclic) properties of a design structure are necessary. The term ,design structure" is used here intentionally because in the case of composite structures, it is not only pure materials (i.e. materials with neglected structural discontinuities) but first of all the joints of concentrated force introductions and glue connections that have the main influence on fatigue life. The load spectra are traditionally presented in the incremental form or in the form of load blocks corresponding to the flight phases or to the flight missions. The load spectra that consist of constant-amplitude load blocks have been used for many decades in fatigue tests. However, such a solution is rather imperfect; for example the crucial problem is the sequence of the load blocks to be applied on the fatigue stand.

The present day form of load spectrum is an array form. It allows not only stochastic load sequence storing, but also reconstructing this sequence in an approximate way [1].

One of the possible characters of load spectrum arrays is, so called, transfer array or half-cycle array. In order to create the transfer array, the load signal is reduced to the form of a local extremes series and divided into the integer number of load levels. (In the figure, for the sake simplicity, there are marked only 12 load levels, but usually 32 load levels are used in fatigue investigations of gliders structure). Then, in the array having the same number of rows and columns as the number of load levels, particular transfers of load signal are recorded and summed. Such an array could be drowned up in ,on line” mood. A special form of transfer array is a half-cycle array. In order to create such an array the algorithm of counting full load cycles needs to be used. The most effective is the Rainflow Counting algorithm. In the case of half-cycle array creation - the whole history of load signal is required. 


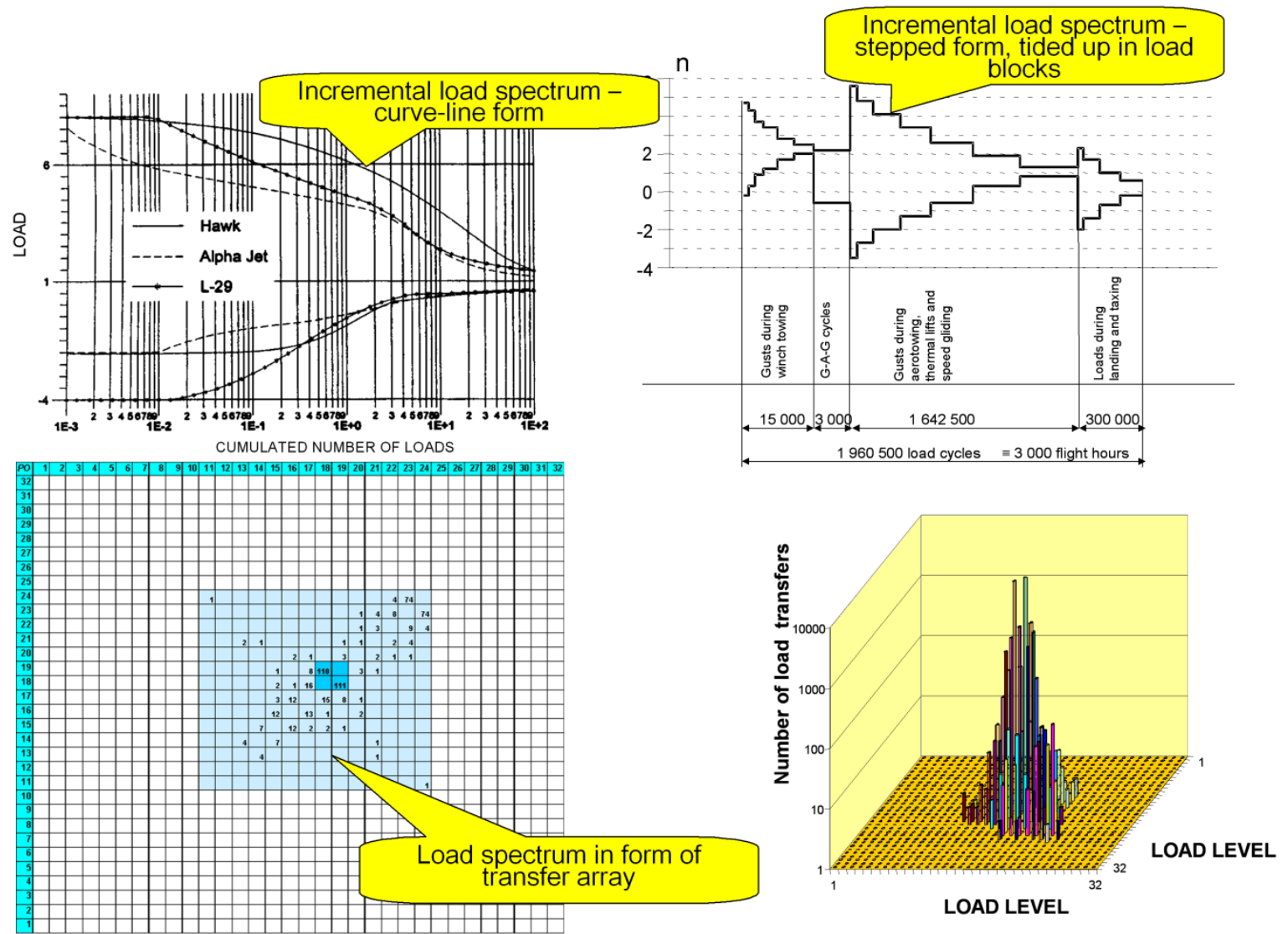

Fig. 1. Different presentation forms of load spectra

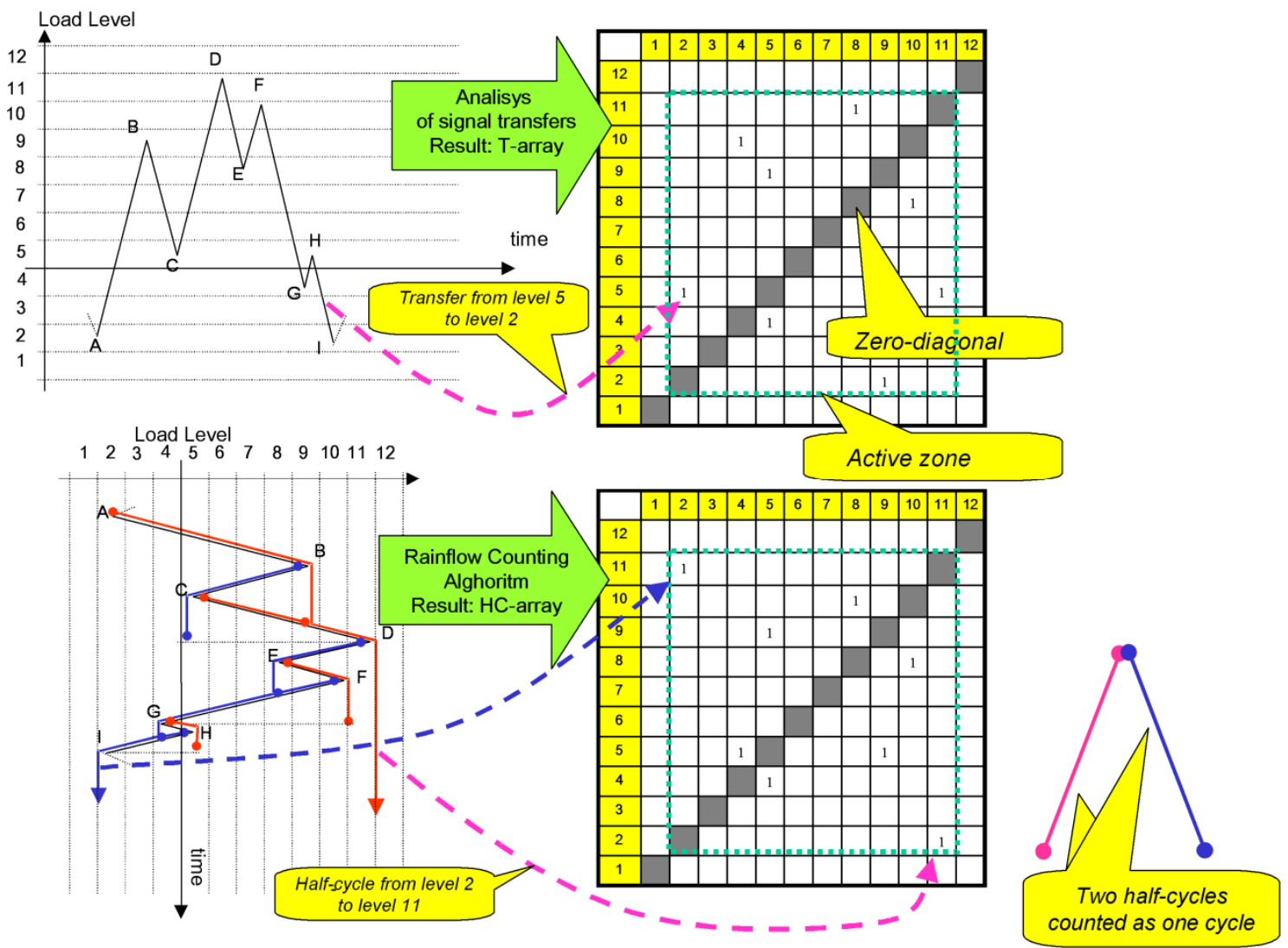

Fig. 2. An array form of load spectra presentation 
As an example there is shown the load spectrum named KoSMOS, which is used for glider certification tests in Germany, and the load spectrum of SZD-31 „Junior” glider, which originally was developed in the form of load blocks, but after conversion to transfer array has the form as in Figure 3. Those load spectra will be used for further comparisons.
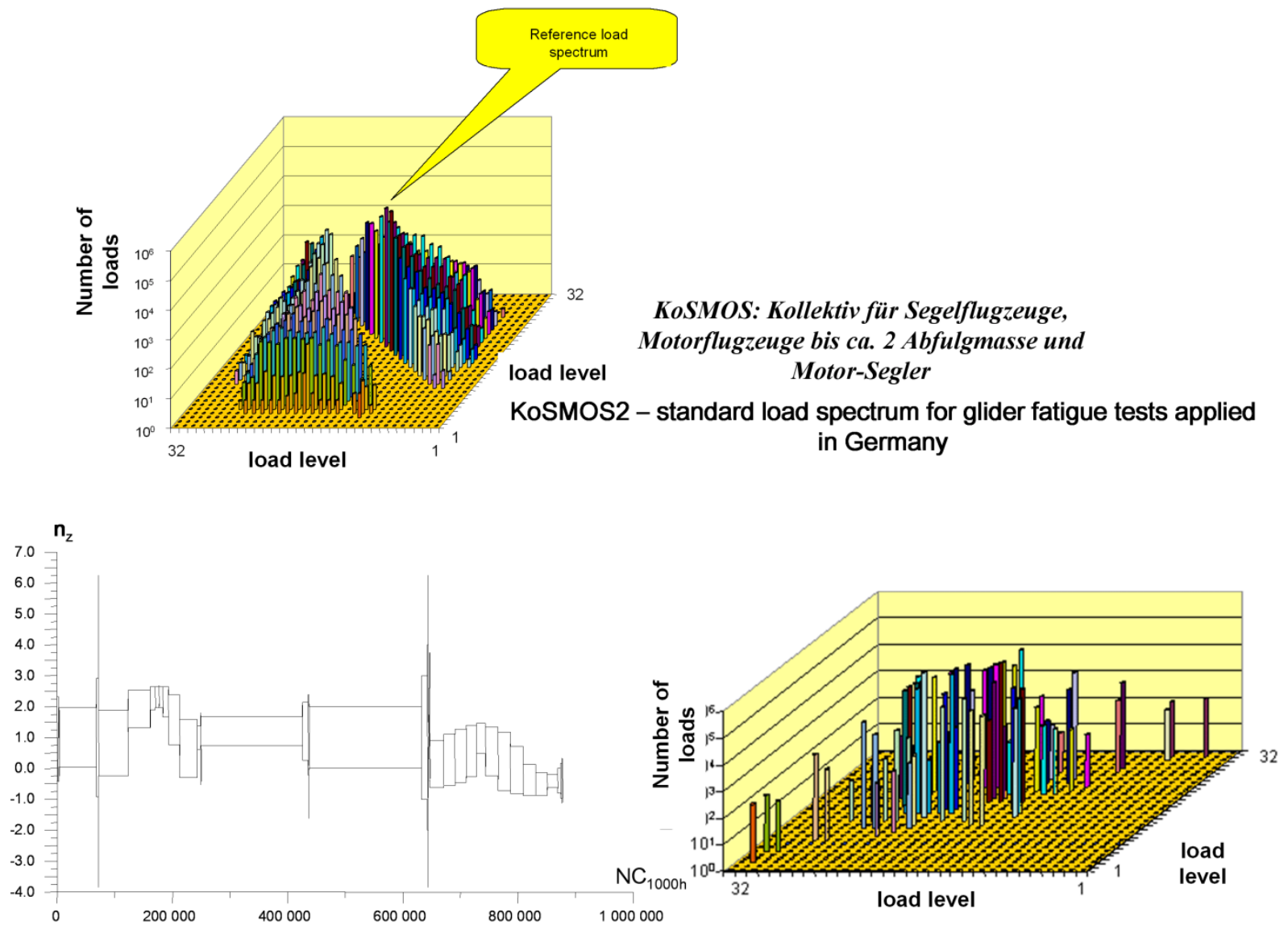

Load spectrumn of SZD-51-1 „Junior” glider (obtained from Stafiej method)

Fig. 3. Reference load spectra

The literature offers a lot of mathematical models of fatigue damage cumulation models, nevertheless, in technical applications, the Palmgren-Minner hypothesis is the most popular even nowadays.

There are several reasons for this state of affairs. Especially:

- Mathematical models of other fatigue hypotheses were created on the basis of testing results of specific specimens (shape, structure versus loading, e.t.c.), which are often very simplified in comparison with the real aircraft structures;

- As universality of most of the fatigue hypotheses is not confirmed, and their application for a particular structure and load state poses the problem of model parameterization, the national aviation authorities generally recognize the Palmgren-Miner hypothesis as applicable for fatigue life proving.

The usefulness of the P-M hypothesis for fatigue damage cumulation during stochastic loads was proven in the literature. Due to the fact that all mathematical models of damage cumulation give approximate results - the aviation authorities assume that there is no point in choosing a more sophisticated hypothesis than the P-M. Imposing the suitable value of the scatter factor compensates for the uncertainty of fatigue damage calculations. It is worth noticing that the P-M hypothesis (with the modification concerning the value of $n / N$ sum) was also applied in the investigations into fatigue life of the wind turbine composite blades. 
The cyclic properties of composite structures necessary for fatigue evaluations are information which is difficult to access. The simplest form of their presentation are S-N curves (Fig. 4). One should be aware that the shape of the S-N curve depends on the value of the cycle asymmetry factor (R-ratio). The range of R-ratio values in the load spectrum of sailplane or aircraft is very large, so it is necessary to have at one's disposal quite a big set of S-N curves. Thus, a much more useful form of information about cyclic properties of the composites is provided by the Haigh or Goodman diagrams.

Another problem to be considered is a big number of specimens which should be tested for fatigue properties of composites determination. This arises from the need to make fatigue investigation for the whole range of R-ratio values, and from the fact that fatigue properties of composites are characterized by large scatter. Those reasons necessitate testing many specimens for each point of the S-N curve, which is necessary for statistical presentation of fatigue life of a particular composite structure.
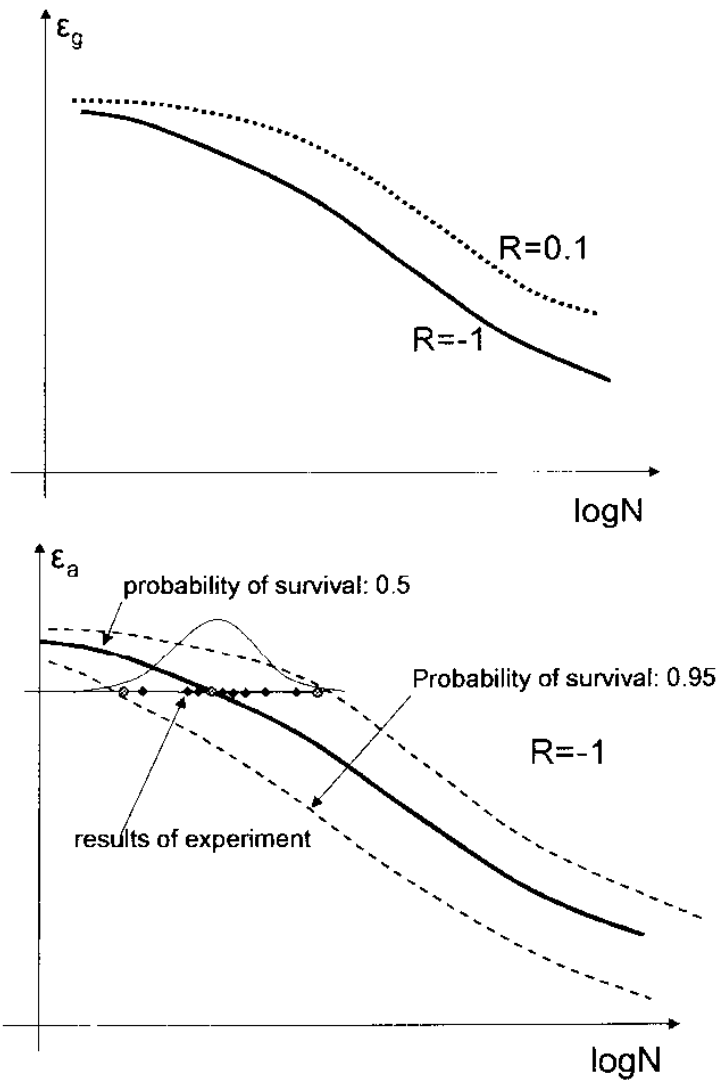

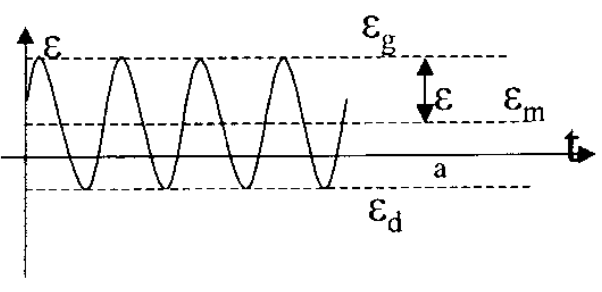

$$
R=\frac{\varepsilon_{d}}{\varepsilon_{g}}
$$$$
R=\frac{\sigma_{d}}{\sigma_{g}}
$$

R-ratio (coefficient of asymmetry)

Fig. 4. Parameters having influence on $S-N$ curves

Figure 5 shows the shape of the load time-courses for chosen points of the Haigh diagram.

While moving along the horizontal line - we deal with the change of the mean value of the load cycle having constant amplitude.

While moving along the vertical line - we deal with the change of amplitude of the load cycle, having constant mean value. 


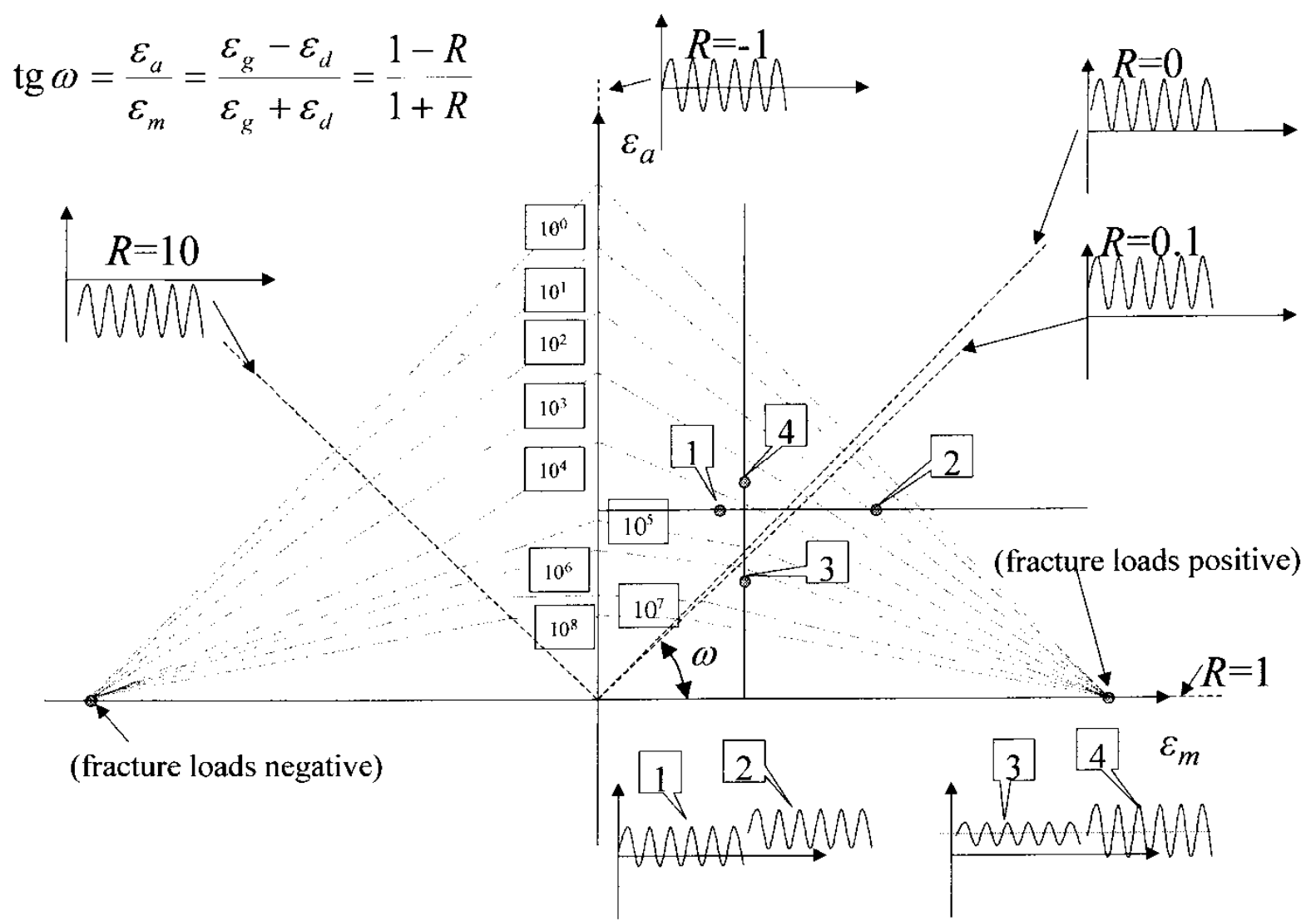

Fig. 5. Correlation between load time-courses and the points on High diagram

Figure 6 presents an example of the Haigh diagram made for the composite used in the wing spar webs applied in some German gliders. It is taken from the article written by Kensche [2], which concerns fatigue evaluations and testing of composite wing spars. This article contains both the High diagrams for composites used in the spar web and in the spar cups. It is worth noticing that such a diagram presents cyclic properties of the composite in an approximate way. It is derived on the basis of fatigue tests results for specific values of R-ratio. The Goodman diagrams are similar to the one presented here, but are drawn up for bigger numbers of R-ratio values.

What draws one's attention is the symmetry visible in the diagram. It is due to the fact that the diagram concerns the composite shell reinforced by fabrics of equal parameters of warp and fill fibers. In such a case, the shear loads applied to the spar web cause that, depending on the load direction, either warp fibers are tensioned while the fill fibers are compressed or the other way around. Thus, the fatigue properties for both directions of shear loads are identical. It occurs in particular for $\mathrm{R}=10$ and $\mathrm{R}=0.1$, which are connected with the direction and values of the cyclic shear load. The High diagrams of the spar cups subjected to cyclic loading along the fiber direction are not symmetric. 

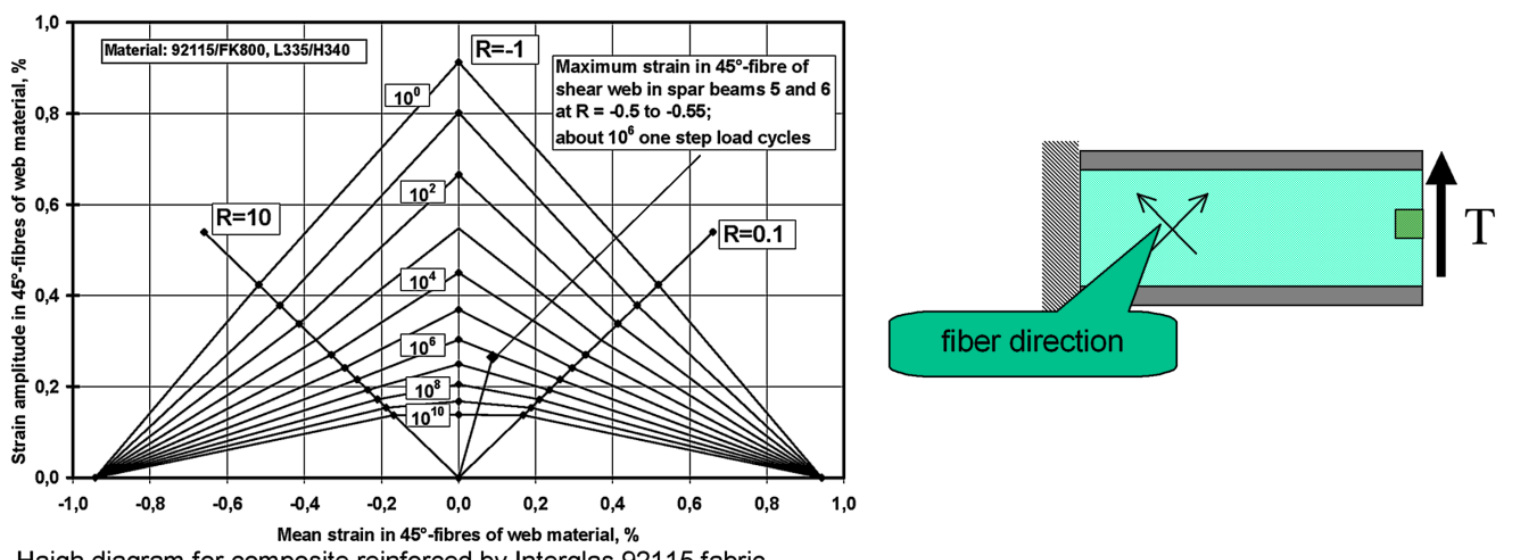

Haigh diagram for composite reinforced by Interglas 92115 fabric

(pure shear loading; diagonal direction of fibers; mean value on number of cycles to failure)

Source: PROPOSAL FOR A CERTIFICATION PROCEDURE OF EXTENDED SAILPLANE LIFETIME Christoph W. Kensche, Institute of Structures and Design, German Aerospace Center (DLR)
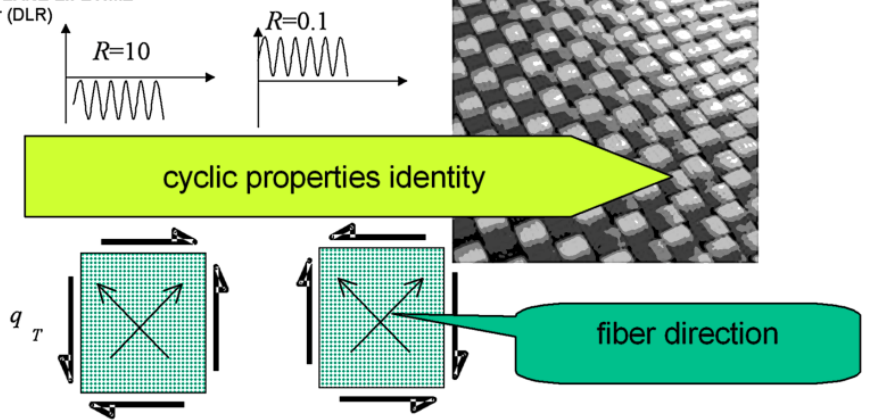

Fig. 6. Example of High diagram for composite wing spar web (reference data)

How many specimens are necessary to be tested to obtain the High diagram?

In the simplest case (i.e. composite shell subjected to shear loading), we approach this as follows: two points corresponding to static strength, at least eight points corresponding to R-ratio equal to -1 , and next eight points corresponding to R-ratio equal to 0.1 (Fig. 7).

In total, we have 18 key points necessary to create the diagram. For statistic evaluations, it is necessary to use at least 7 specimens at each key point. So it means that it is necessary to use the minimum of 126 specimens. Time of specimen fatigue testing depends on the load level and varies between hours and months. Those numbers make it easier to understand why the information about fatigue properties of the composite structure is limited and difficult to be found.

The biggest data-base comes from investigations performed during realization of big EU projects focused on developing wind power plants, for example the project OPTIMAT BLADES, which was summarized in the report by Krause and Kensche published in 2006 under the title "Summary Fatigue Test Report OB_TG1_R026 rev. 0" (unfortunately with the note "Confidential"). Prior to that, a similar report "DOE/MSU Composite Material Fatigue Database: Test Methods, Materials, and Analysis" - CONTRACTOR REPORT SAND97-3002, UC-1210 was published by Mandell and Samborsky in the USA in 1997. Inside, it is possible to find a number of S-N curves and Goodman diagrams for some composite structures. 


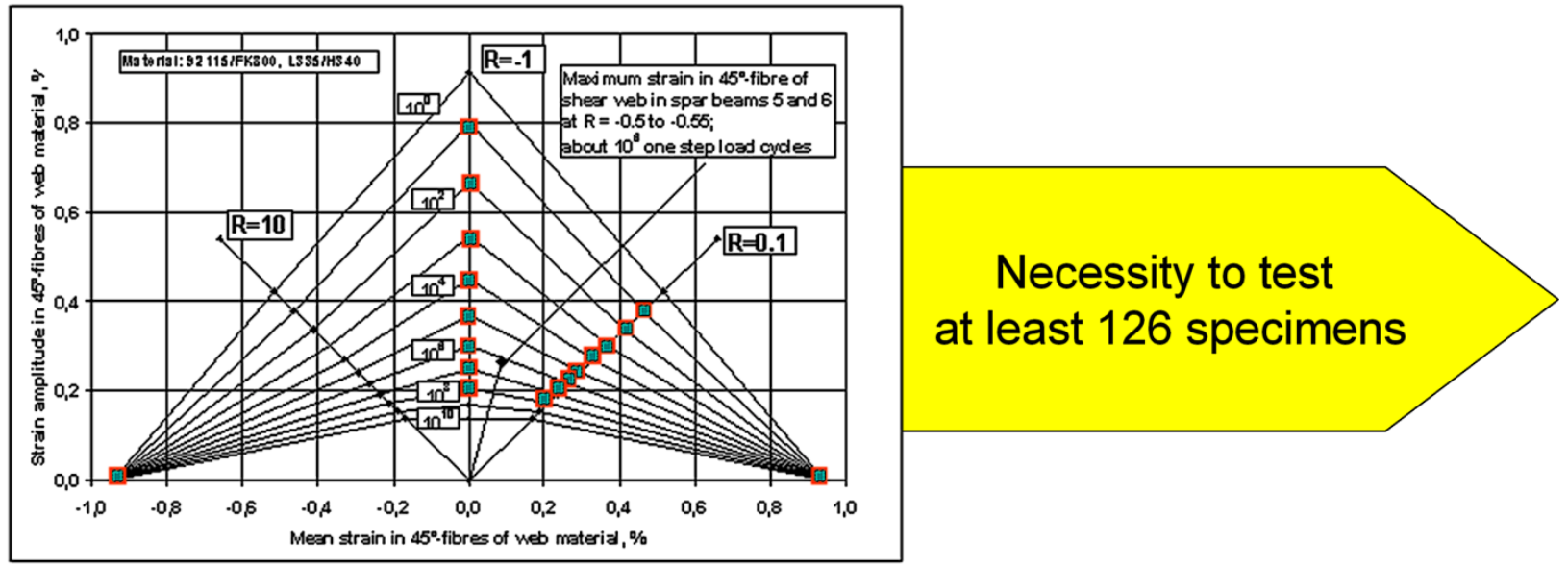

Fig. 7. Key points for High diagram determination

A very important feature for fatigue calculations is the topographic similarity of the High diagram and the transfer array. In the upper part of Figure 8 there is presented a High diagram with the axis oriented in the way corresponding to the organization of the transfer array, which is marked in beige. The beige area envelops the whole range of load-bearing capacity of the material. The operational range of loads is marked in green and divided into the standard number of load levels.

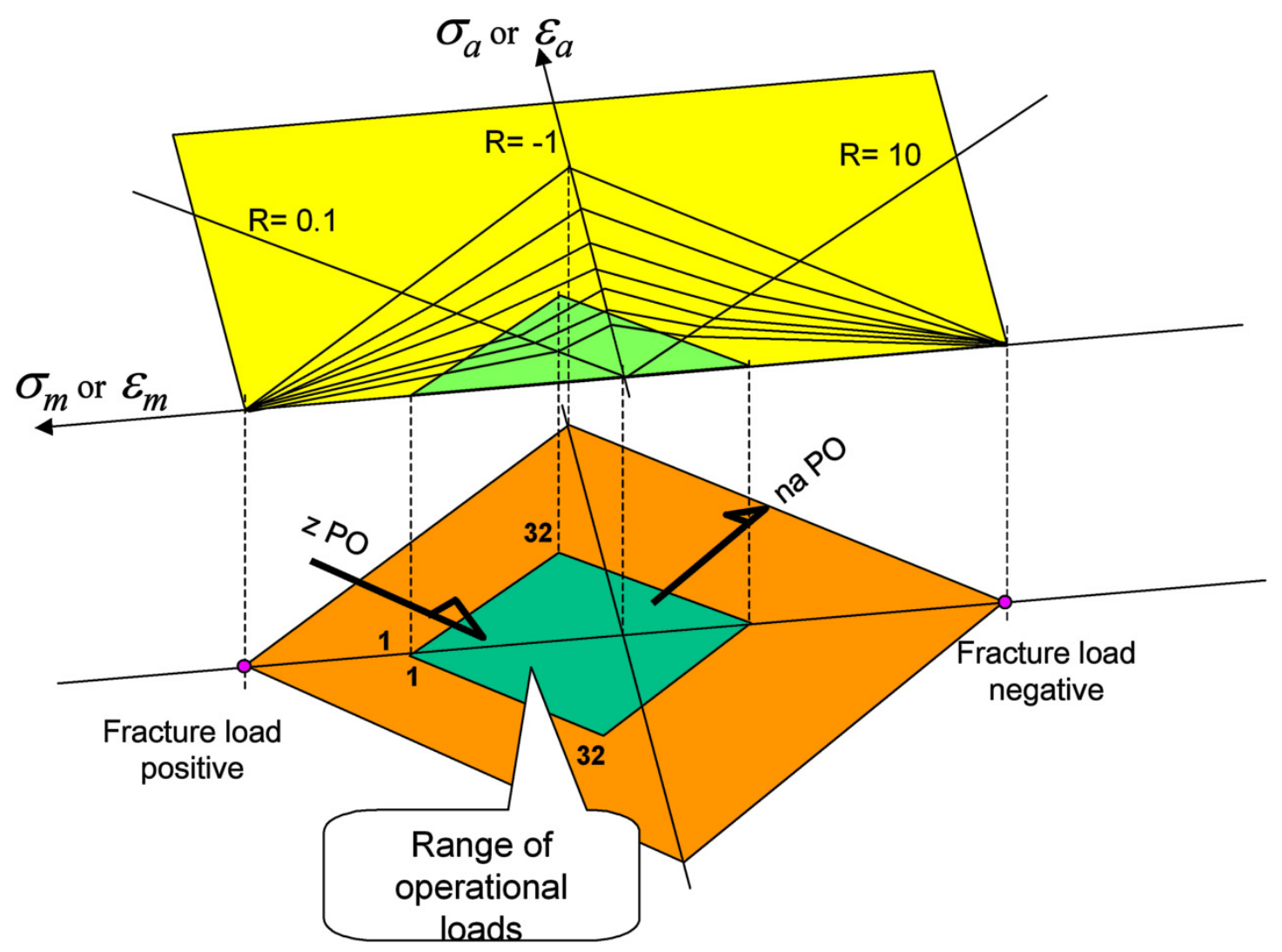

Fig. 8. Topographic similarity of a High diagram and a transfer array

This similarity allows presenting the cyclic properties of the material (or of the structure) in the shape of arrays which are similar to the transfer arrays. Figure 9 presents the number of cycles to failure, marked in the High diagram in green. This array was obtained by calculating the number of cycles to failure for each possible change of the load signal from level $i$ to level $j$. 
To be able to perform this operation, the original High diagram was substituted with a virtual surface, where the vertical coordinate describes the logarithm of a number of cycles to failure. The surface was obtained by approximation and extrapolation of the data contained in the original High diagram. With the load spectrum in the form of transfer array and the array of number of cycles to failure, it is very easy to calculate the fatigue damage value $\mathrm{D}$, by using a modified formula for the P-M hypothesis.

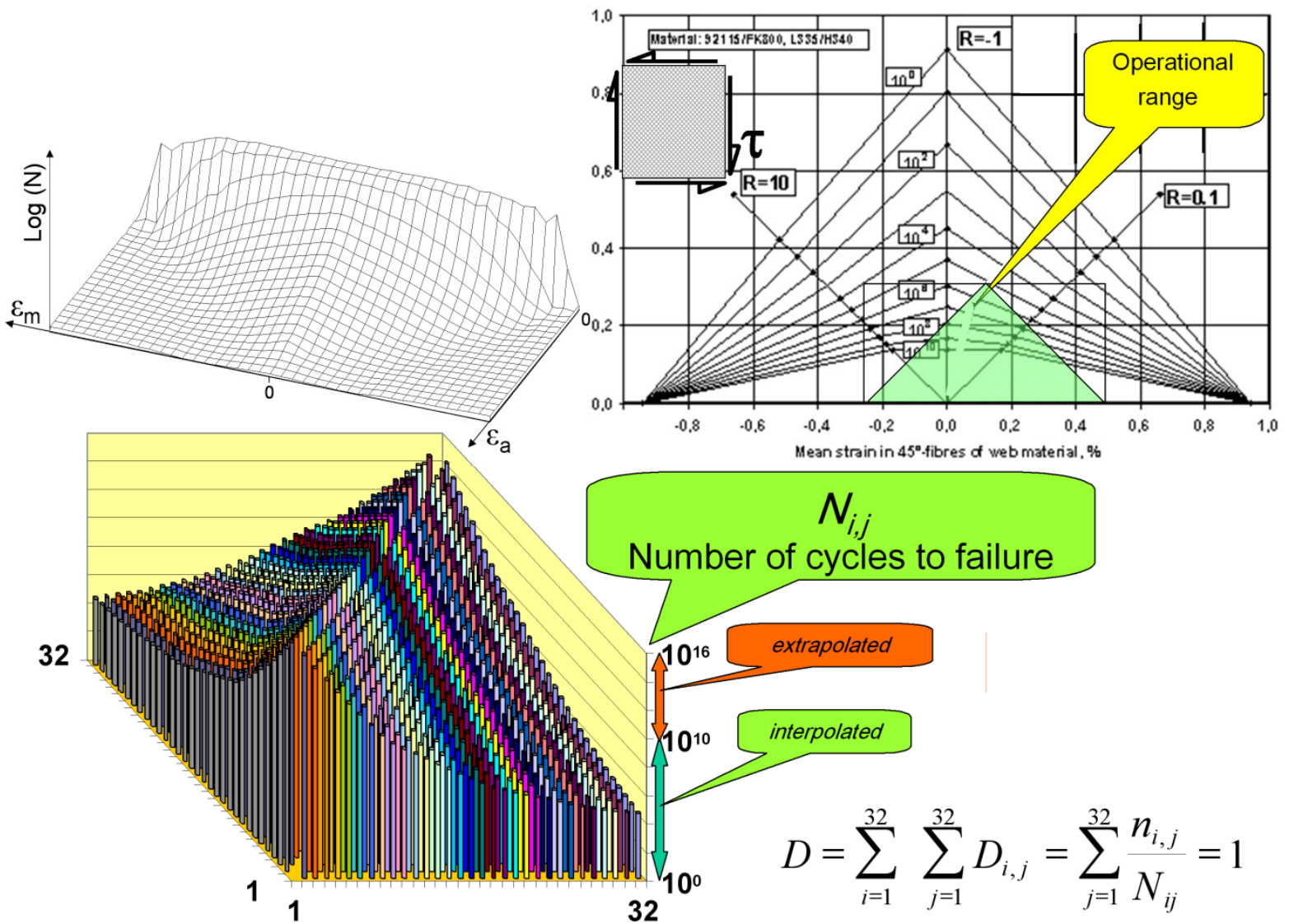

Fig. 9. Array form of material fatigue properties presentation

The simplified methodology of the fatigue properties estimation relies on the estimation of the virtual surface, which describes in an approximate way the number of cycles to failure in the operational zone of loads in the High diagram. This estimation is based on the results of four series of fatigue tests [3].

The $1^{\text {st }}$ step of the simplified procedure of the fatigue properties investigation consists in the determination of the threshold value of the material fatigue sensitivity for $R=-1$. It is represented by point $\mathrm{A}$ in Figure 10, and it could be interpreted as the loads that cause a noticeable drop in the residual strength after $10^{4}$ cycles.

The $2^{\text {nd }}$ step consists in the determination of loads which exert the same influence on the residual strength after $10^{4}$ cycles at the point $\mathrm{A}$ as at point $\mathrm{B}$, which lies on the line representing $R$ $=0.1$ (and as at point $\mathrm{C}$ if necessary), and then as at point $\mathrm{D}$. It is worth emphasising that points $\mathrm{B}$, $\mathrm{C}, \mathrm{D}$ are situated along a line of constant load amplitude, which facilitates the fatigue testing.

The $3^{\text {rd }}$ step consists in spreading the virtual plane based on points A,B,D. It is assumed that the vertical coordinate represents the logarithms of cycles to failure.

The $4^{\text {th }}$ step of this procedure consists in the determination of the number of cycles to failure in points $\mathrm{E}, \mathrm{F}$, and $\mathrm{G}$, which belongs to the plane $\mathrm{ABD}$. 


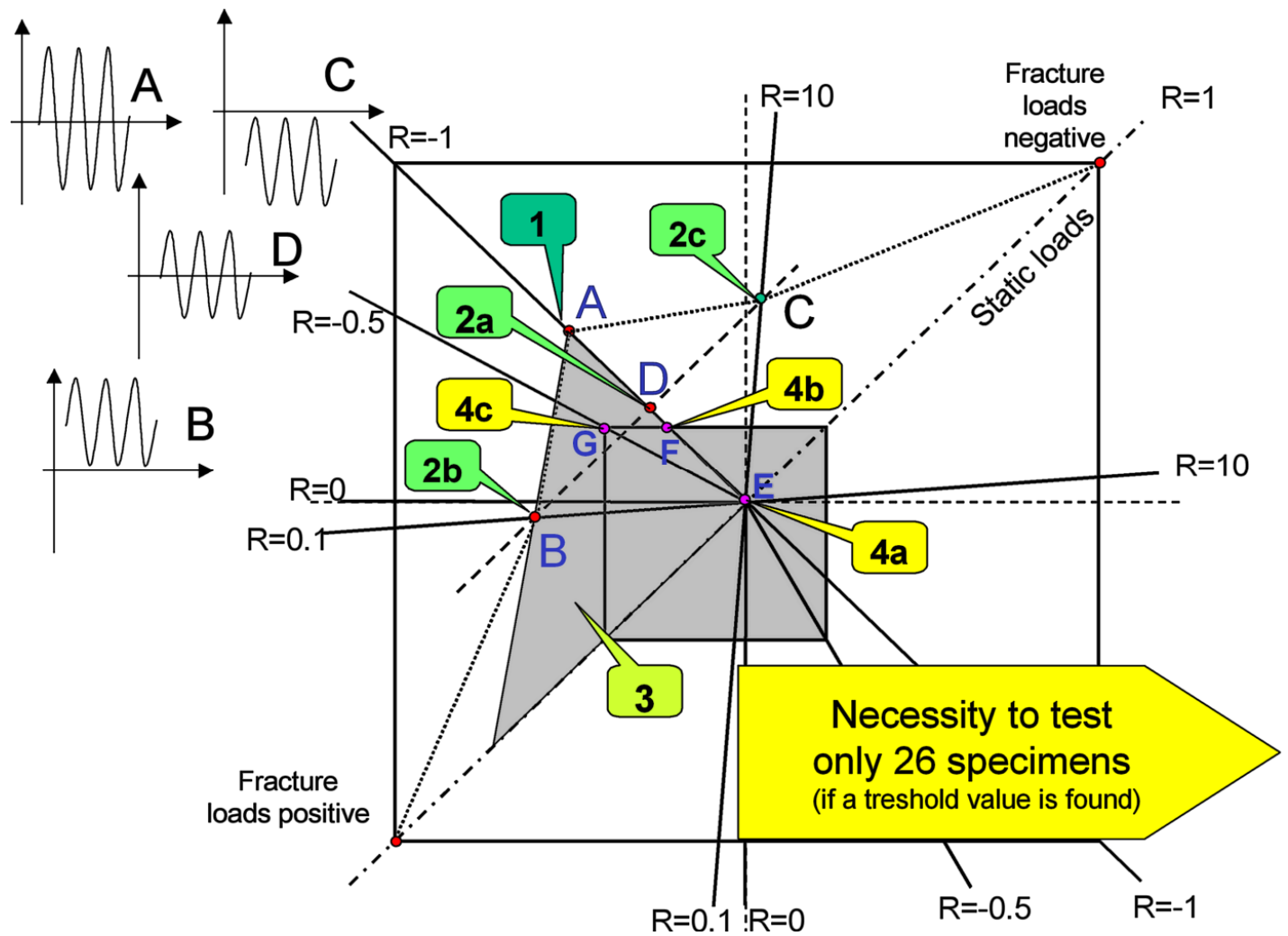

Fig. 10. Key points for simplified method of fatigue properties estimation for composite structures

In the last (i.e. $5^{\text {th }}$ ) step of the procedure there are created two virtual planes E'GF and E'FE', which make up the surface representing the number of cycles to failure in the zone of operational loads (Fig. 11).

It was assumed that the number of cycles to failure is constant along the 0-diagonal and is the same as at point $\mathrm{E}$.

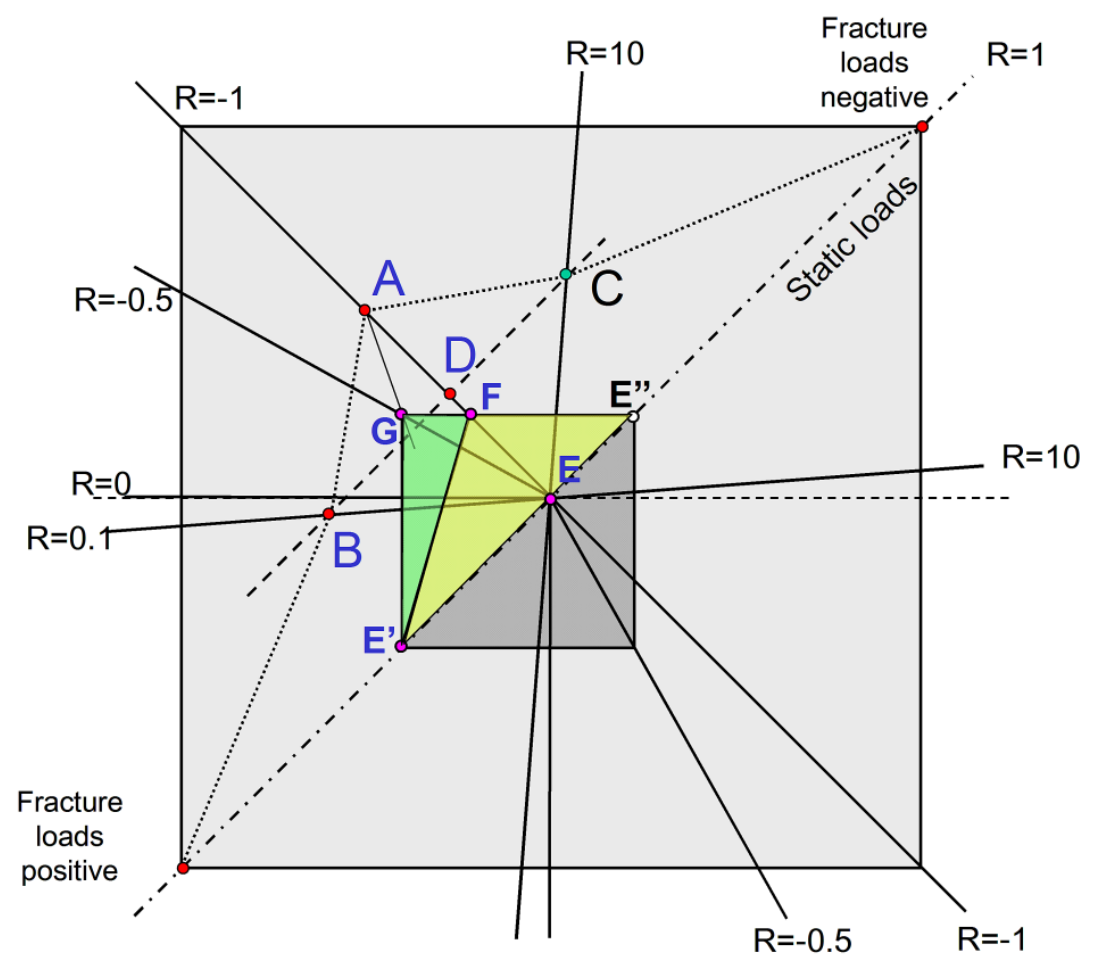

Fig. 11. Virtual surface approximating fatigue properties of composite structures 
Figure 12 represents in 3D the array of cycles to failure, which was derived following the above described procedure. For the purpose of comparison, the array of cycles to failure based on the Haigh diagram taken from Kensche's article was also shown. There are visible significant differences in the zones close to the 0-diagonal of both arrays. But it is worth noticing that: a/ those differences concern the extrapolated zone in relation to the original Haigh diagram, so the real error is impossible to determine; $\mathrm{b} /$ for composites, the cycles near the 0-diagonal in the transfer arrays have insignificant influence on the damage cumulation value D.

\section{Result of interpolation/approximation of the data from High diagram for composite shell under shear loads}

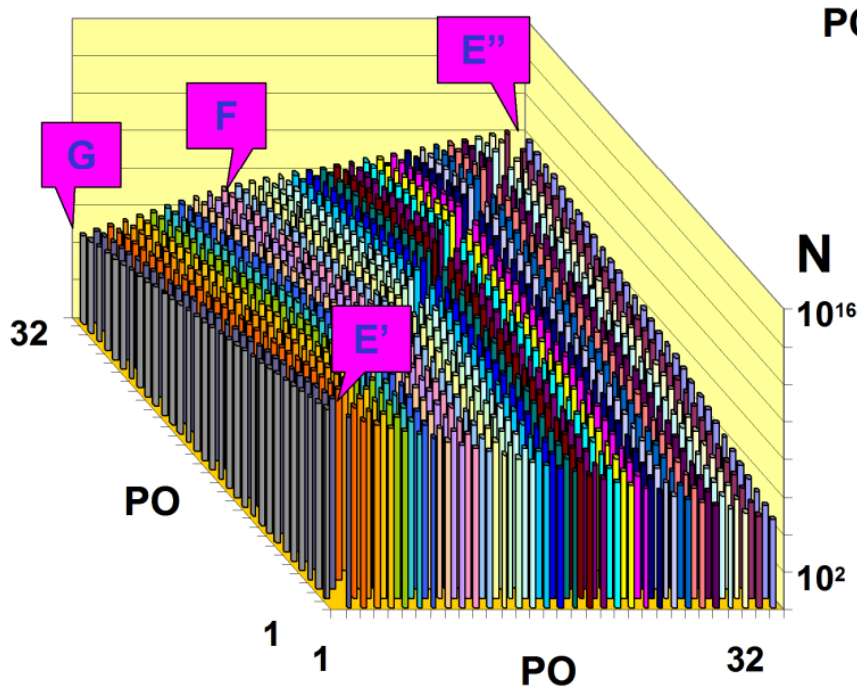

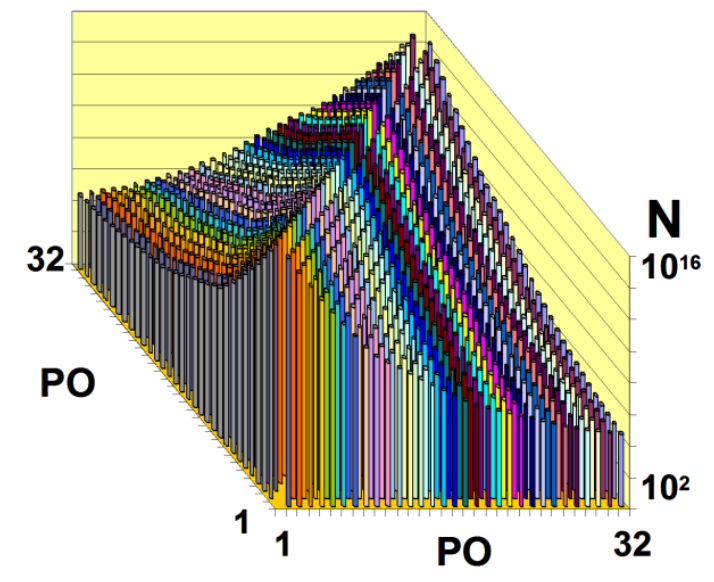

Result of simplified method

Fig. 12. Comparison of fatigue data derived from High diagram and by simplified method

Figure 13 presents the D-values, derived for two already presented reference load spectra, made for the fatigue data obtained from the High diagram and for the fatigue data achieved from the procedure described here. For the load spectrum KoSMOS2 - the differences between D values are negligible. For the load spectrum STAFIEJ, the difference is quite big (about 35\%), however, it is still lower than the difference between D values for both spectra calculated based on the High diagram, and, more importantly, the application of the simplified procedure causes reduction of this difference. The error resulting from the application of the simplified procedure loses its significance when we realize that in the case of KoSMOS2 the D value presented in the figure refers to 6000 flying hours, while in the case of STAFIEJ - it refers to 1000 flying hours (because for such values those load spectra are drawn up.

We can conclude that the simplified methodology of the fatigue properties estimation for polymer composite structures, although is not quite perfect, seems to be reasonable. Future experimental investigations will verify the expectation related to the described method. 


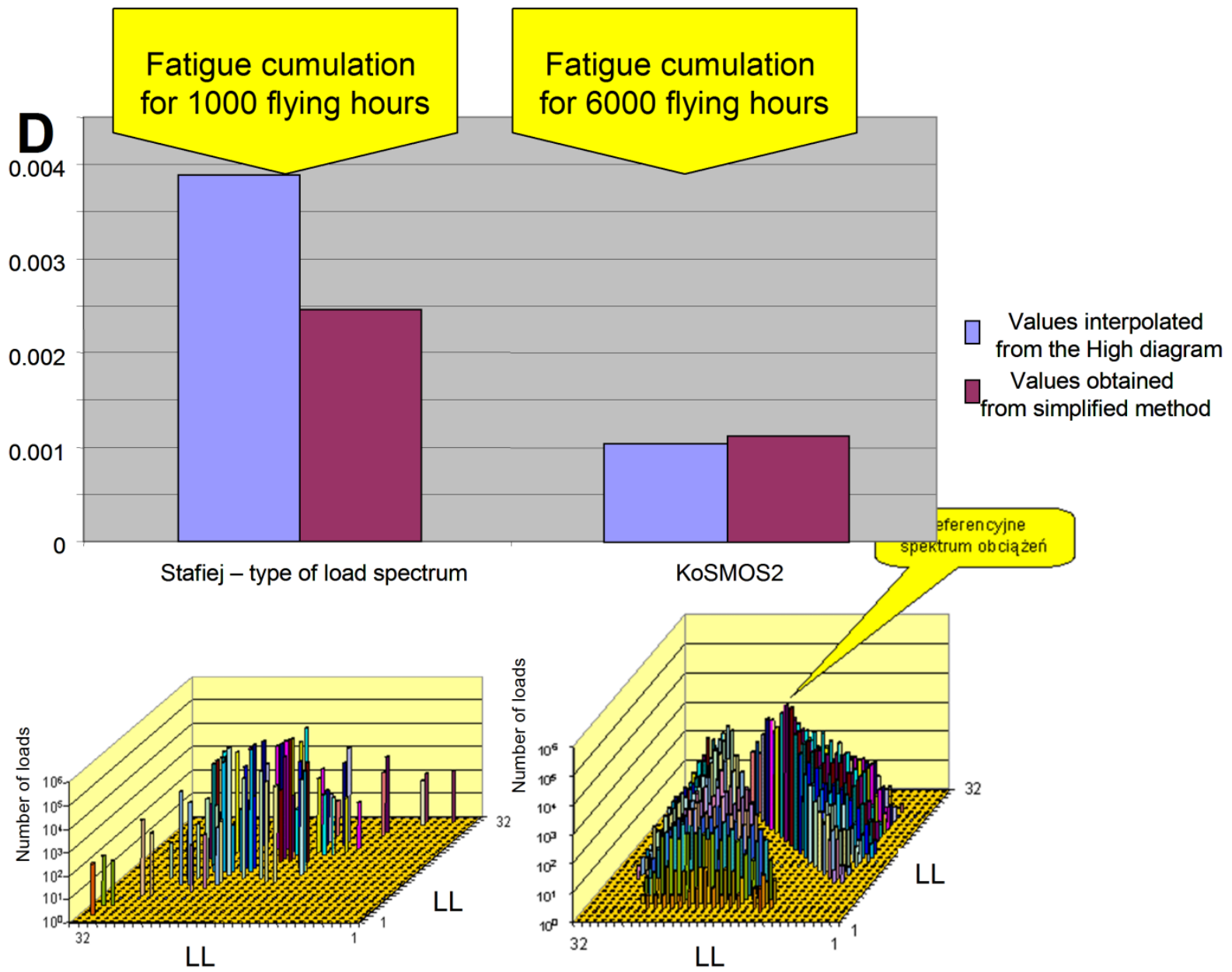

Fig. 13. Comparison of D-values calculated for the data taken from High diagram and simplified method

\section{REFERENCES}

[1] Rodzewicz, M. (2008). Determination and extrapolation of the glider load spectra. Aircraft Engineering and Aerospace Technology, 80(5), pp. 487-496.

[2] Kensche, C., W. (2002). Proposal for a certification procedure of extended sailplane lifetime. Technical Soaring, 26(2), pp. 32-43.

[3] Rodzewicz, M. (2009). Fatigue evaluation of high-loaded aeronautical composite structures. Aircraft Engineering and Aerospace Technology, 81(5), pp. 452 - 460. 